\title{
Modeling asymmetric cavity collapse with plasma equations of state
}

\author{
Brett Tully ${ }^{*}$ and Nicholas Hawker \\ First Light Fusion Ltd., Begbroke Science Park, OX5 1PF, United Kingdom \\ and Department of Engineering Science, University of Oxford, OX1 3PJ, United Kingdom
}

Yiannis Ventikos

Department of Mechanical Engineering, University College London, Torrington Place, Cordon, WC1E 7JE, United Kingdom

(Received 19 November 2013; revised manuscript received 29 February 2016; published 12 May 2016)

\begin{abstract}
We explore the effect that equation of state (EOS) thermodynamics has on shock-driven cavity-collapse processes. We account for full, multidimensional, unsteady hydrodynamics and incorporate a range of relevant EOSs (polytropic, QEOS-type, and SESAME). In doing so, we show that simplified analytic EOSs, like ideal gas, capture certain critical parameters of the collapse such as velocity of the main transverse jet and pressure at jet strike, while also providing a good representation of overall trends. However, more sophisticated EOSs yield different and more relevant estimates of temperature and density, especially for higher incident shock strengths. We model incident shocks ranging from 0.1 to $1000 \mathrm{GPa}$, the latter being of interest in investigating the warm dense matter regime for which experimental and theoretical EOS data are difficult to obtain. At certain shock strengths, there is a factor of two difference in predicted density between QEOS-type and SESAME EOS, indicating cavity collapse as an experimental method for exploring EOS in this range.
\end{abstract}

DOI: 10.1103/PhysRevE.93.053105

\section{INTRODUCTION}

The inertial collapse of a gas cavity suspended in a liquid can result in a significant focusing of energy. Among many examples, cavity collapse is known to destroy ship propellers [1] and can be used to sensitize explosives [2]. More relevant to the present work is the observation of bright flashes of light from collapsing cavities [3-7]. The most widely researched example of this phenomenon is single-bubble sonoluminescence (SBSL), where a single gas cavity is ultrasonically driven to stably oscillate between $\sim 1$ to $50 \mu \mathrm{m}$ radius [4]. SBSL is a resonant phenomenon with a limited parameter space that places an upper bound on the achievable energy density at minimum volume [8,9]. Recent work examines a similar but nonperiodic spherical collapse process which avoids the limitations of SBSL and achieves significantly higher energy densities [10].

Asymmetric cavity collapse - characterized by the formation of a main transverse jet [11] — is a hydrodynamically very different process to SBSL that can achieve a different set of thermodynamic conditions. Intensification provided through spherical symmetry is sacrificed, yet the collapse maintains various significant energy focusing processes. Previous efforts by the current authors examine the detailed asymmetric collapse of a polytropic air cavity in water when struck by a planar shock wave across a range of intensities. The incident shock was varied from 0.1 to $100 \mathrm{GPa}$ and peak densities and temperatures in the gas reached conditions in the order of $1 \mathrm{~g} / \mathrm{cm}^{3}$ and $10 \mathrm{eV}$ [11]. The hydrodynamics of the simulated collapse processes showed excellent agreement with available experimental literature; however, the achieved conditions exceed the validity of the ideal gas equation of state (EOS). In the current work we replace air with argon

*brett.tully@firstlightfusion.com and compare and contrast the ideal gas EOS with more sophisticated, realistic, and detailed plasma modeling.

\section{METHODOLOGY}

\section{A. Numerical method}

The hydrodynamics of shock-cavity interaction are a strong challenge to computational fluid dynamics methodologies regardless of the EOS employed; indeed, the problem has been used as a benchmark [12,13]. The method must solve Euler's equations of compressible flow and in doing so must adequately resolve the interface between the liquid and the gas as well as the various strong emergent shock waves. Modern Godunov-type schemes provide sufficient accuracy for the shock waves; however, numerical diffusion over the gas-liquid contact smears this all-important interface and an explicit treatment is required. The volume of fluid method [14] and the level set method [15] are two schemes often applied, however, front-tracking approaches compare favorably in a number of aspects (see section 4 of Ref.[16]).

Front-tracking resolves the gas-liquid interface through the use of a two-grid methodology; a Lagrangian hypersurface is used to represent the interface and is overlaid on an Eulerian grid upon which the bulk flow can be solved. The hypersurface is comprised of a collection of lines (two dimensional, or 2D) or triangles (three dimensional, or 3D) where each vertex holds the thermodynamic state for the fluid on either side of the hypersurface. Per time step, the two states at each vertex are updated by solving Riemann problems in the normal and tangential directions, while the location of the vertex is updated via propagation in the normal direction. Over time the vertices will converge and diverge, and potentially overlap, so the method must take care to untangle and/or redistribute the hypersurface [16-18].

Finally, the updated hypersurface defines a set of boundary conditions for the update of states on the Eulerian grid. Any 
numerical diffusion over the gas-liquid interface is eliminated and the coupling between adjacent fluid bodies is encapsulated within the normal propagation stage. If the Riemann problem can be solved, entirely distinct physics, e.g., different EOS, can be modelled in each region, a strong advantage of front tracking. We employ the van Leer MUSCL solver $[19,20]$ and the Riemann solver is an implementation of the exact solver [21].

\section{B. Equation of state}

For the water component, we use a stiffened polytropic EOS [22] with constants found by fitting the experimental data for the principal Hugoniot of water and the IAPWS-95 equations of state [11]. For argon, we compare three families of EOSs: the ideal gas equation, tables generated based on the quotidian equation of state(QEOS) [23], and a table from the SESAME database [24].

The QEOS family of equations calculate the thermodynamic functions from the Helmholtz free energy, with the electronic contribution determined from the Thomas-Fermi (TF) model [25] and ionic contributions from the Cowan model [23]. The TF cold curve is improved through a softsphere function, giving a better estimate of the critical pressure and critical temperature [26-28]. We use one table generated by the Lawrence Livermore National Laboratory (LEOS), and a second from Goethe University in Frankfurt (FEOS). The FEOS table is generated by using values for the reference state $\left(T_{\text {ref }}=82.3 \mathrm{~K}\right.$ and $B=\frac{1}{3}\left(C_{11}+2 C_{12}\right)=1.83 \mathrm{GPa}$ [29]), cohesive energy $\left(E_{\text {coh }}=7.74 \mathrm{~kJ} / \mathrm{mol}\right.$ [30]), and soft-sphere values ( $m=0.9$ and $n=4.0$ [31]).

As documented and described in the notes of the table, SESAME 5172 incorporates the physics of six different theoretical models [32]: (i) a Saha model for the ideal gas, ideal plasma, and part of the ionization equilibrium regime; (ii) a nonideal, quantum-statistical-mechanical theory for the remaining ionization equilibrium regime; (iii) a ThomasFermi-Kirzhnits model corrected for thermal ion contributions in compression [33]; (iv) electron band theory embodied in a linear muffin-tin orbital model for the cold curve [34]; (v) an exponential six-fluid perturbation theory for the high-density fluid [35]; and (vi) an analytic fit to the Lennard-Jones potential for the neutral fluid region [36].

The gaseous principal Hugoniot for each EOS is presented in Figure 1, along with experimental results from Christian et al. [37]. The QEOS-type EOSs show a smooth departure from ideal gas conditions. However, at lower pressures SESAME corresponds closely with ideal gas, diverging around $10 \mathrm{MPa}$, with the density values matching well to reported experimental values. As the conditions intensify, SESAME and QEOS converge in their predictions; Carpenter et al. discuss the transition and differences in greater detail [38]. It should be noted that, generally, compared to ideal gas, plasma EOSs predict greater compression and lower temperature for a given shock strength because ionization is an energy sink.

\section{Thermodynamic functions}

To solve for the conservation equations, the Riemann solver requires the following fundamental state variables to be provided: density $\rho$, temperature $T$, pressure $P$, specific internal energy $E$, specific entropy $S$, specific heat at constant

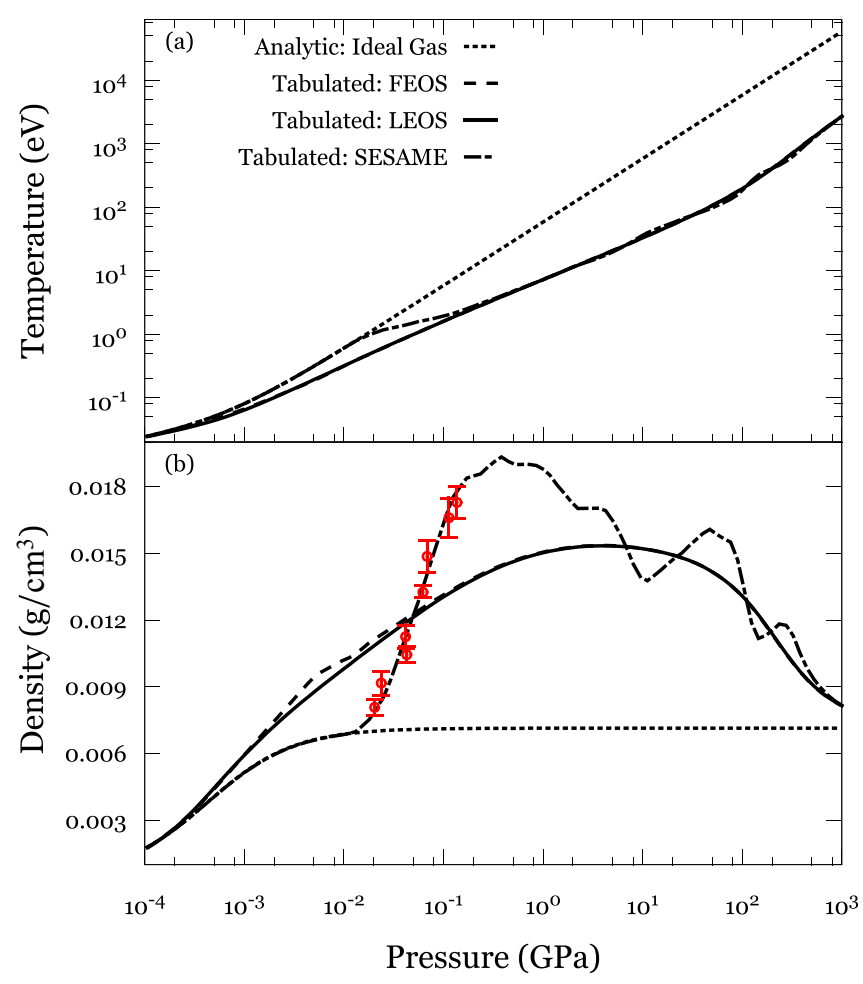

FIG. 1. Principal Hugoniot for $\operatorname{argon}\left(T_{0}=285 \mathrm{~K}, P_{0}=1 \mathrm{bar}\right)$. Experimental data (red dots) from Christian et al. [37] as presented in Carpenter et al. [38]. The match of SESAME to experimental data suggests that, in contrast to the QEOS, this EOS has specifically incorporated principle Hugoniot studies into its model.

volume $c_{v}=\left.\cdot \frac{\partial E}{\partial T}\right|_{\rho}>0$, Grüneisen parameter $\left(\Gamma=\left.\frac{1}{\rho c_{v}} \cdot \frac{\partial P}{\partial T}\right|_{\rho}\right)$, and thermodynamically consistent sound speed squared $\left(c^{2}=\right.$ $\left.\cdot \frac{\partial P}{\partial \rho}\right|_{T}+\left.\frac{1}{\rho} T \Gamma \cdot \frac{\partial P}{\partial T}\right|_{\rho} \geqslant 0$ [39]). In addition, the numerical methodology requires a number of EOS-specific calculations outlined below. With analytical EOS, these functions can be derived explicitly (see Ref. [21] among others); for general EOS they must be solved numerically.

For a wave of given pressure $P_{1}$ traveling into a known ahead state $\left(\rho_{0}, P_{0}\right)$ we must be able to calculate the density behind the wave $\left(\rho_{1}\right)$. In the case of a shock wave $\left(P_{1}>P_{0}\right)$, the density is given by the Hugoniot relationship: $E_{1}=E_{0}^{\prime}-$ $\frac{\bar{P}}{\rho_{1}}$ where $E_{0}^{\prime}=E_{0}+\frac{\bar{P}}{\rho_{0}}$ and $\bar{P}=\frac{1}{2}\left(P_{0}+P_{1}\right)$. This equation can be solved using a simple root-finding algorithm, where the lower bound on the density is given by the ahead state and the upper bound can be set by assuming that a single shock wave provides a maximum compression ratio. The root-finding process requires that the sign of $\left.\frac{\partial E}{\partial \rho}\right|_{P}$ is constant through the range of densities. An initial guess for $\rho_{1}$ can be calculated by fitting a stiffened polytropic equation of state to the ahead state and solving its Hugoniot equation such that: $\quad E_{1}=\bar{P}\left(P_{1}-P_{0}\right)\left[\left(\rho_{0} c_{0}\right)^{2}+\frac{1}{2} \rho_{0}\left(P_{1}-P_{0}\right)\left(\Gamma_{0}+2\right)\right]^{-1}$ (obtained from Refs. [22,40]). In the case of a rarefaction wave $\left(P_{1}<P_{0}, S_{0}=S_{1}\right)$, the density can be calculated by root finding the EOS relationship for entropy as a function of density and pressure where the lower bound of density is given as the vacuum density $\left(10^{-28} \mathrm{~g} / \mathrm{cm}^{3}\right)$ and the upper bound is 
the value of the ahead state. In the case of tabulated EOS, performance optimization can be achieved by precomputing a table of $\rho(P, S)$ to allow direct interpolation for density.

The wave curve $w$ defines a locus of all possible states that can be connected to the ahead state through either a rarefaction wave (expansion branch) or a shock wave (compression branch) and plays a crucial role in the solution of a Riemann problem [22]. Given the pressure jump across the wave, the wave curve can be formulated in terms of the change in particle speed and is intimately linked to the mass flux across the wave $(m)$, such that $w=u_{1}-u_{0}= \pm \frac{1}{m}\left(P_{1}-P_{0}\right)$ where the \pm defines the direction of the wave (Eq. 3.36 in Ref. [22]). The mass flux is the shock propagation speed in Lagrangian coordinates. If there is no pressure gradient, the change in particle speed is zero and the mass flux is given by the impedance $(z=\rho c)$ of the ahead state; for a shock wave, $w=\left[\left(P_{1}-P_{0}\right)\left(\frac{1}{\rho_{0}}-\frac{1}{\rho_{1}}\right)\right]^{1 / 2}$, with $\rho_{1}$ given by the Hugoniot relationship as described above; and for a rarefaction wave $w=I_{d p}=\left.\int_{P_{1}}^{P_{0}} \cdot \frac{d P}{\rho c}\right|_{S}$. Finally, the iterative procedure of the Riemann solver often tests for a vacuum mid-state by calculating the maximum possible velocity of the left wave, $u_{l \max }=u_{l}+w_{\mathrm{vac}, l}$, and the minimum possible velocity of the right wave, $u_{r \min }=u_{r}-w_{\mathrm{vac}, r}$; if $u_{l \max } \geqslant u_{r \min }$, the mid state is considered to be a vacuum (see Fig. 4(e) of Ref. [22]). Calculating the wave curve for the vacuum pressure $\left(10^{-16} \mathrm{~Pa}\right)$ is challenging for tabulated EOSs, as for all but the lowest entropies the vacuum pressure is unlikely to be contained in the table; we overcome this by precomputing a table of $I_{d p}(P, S)$ that can be interpolated directly, while also defining a minimum pressure bound for given values of entropy to prevent extrapolation from this table.

\section{Table interpolation}

Historically, interpolation is used at run-time to evaluate $E$, $P, .\left.\frac{\partial E}{\partial \rho}\right|_{T},\left.\cdot \frac{\partial P}{\partial \rho}\right|_{T}, .\left.\frac{\partial E}{\partial T}\right|_{\rho}$, and.$\left.\frac{\partial P}{\partial T}\right|_{\rho}$ for any given $(\rho, T)$ pair, where the derivatives are calculated from the interpolating function. These are used as building blocks for evaluating the remaining fundamental state variables [24,41]. This approach was developed with a focus on conserving memory usage at the cost of CPU compute time; modern computing hardware, however, allows us to develop an alternative scheme where we precompute the fundamental state variables on a dense density-temperature grid. This allows arbitrary order in the finite difference methods for computing the derivatives and then, by storing the resulting state variables, a simple bilinear interpolation can be used at runtime without concern for derivatives being continuous across grid points. Higher-order interpolation schemes such as BIMOND $[42,43]$ can be introduced at the precompute stage to up-sample the table density, increasing accuracy without significant impact on runtime.

Furthermore, the numerical method requires three representations of state- $(\rho, T),(\rho, P)$, and $(\rho, E)$ - that are used interchangeably throughout the different algorithms. Thus, the interpolation library must provide a mapping between each of the pairs. The most basic form of this inverse interpolation is to calculate temperature given density and pressure or energy, while transferring between pressure and energy is achieved by using the temperature as a pivot, further details can be found in Ref. [44]. Finally, for certain isentropic processes mentioned above, significant performance gains were achieved through precomputing pressure-entropy grids of $\rho(P, S)$ and $I_{d p}(P, S)$.

\section{Model setup}

We model a single argon cavity, of $5 \mathrm{~mm}$ radius, in water with initial conditions of $P_{0}=101.3 \mathrm{kPa}, T_{0}=300 \mathrm{~K}$ in two dimensions [see Fig. 2(a)]. The Eulerian mesh is defined with 250 cells per radius length, satisfying grid-independence tests [44]. Previous studies have shown sufficient similarity between two- and three-dimensional simulations, thus justifying the use of two dimensions in this work $[11,45]$. Important features of the collapse are formation of the main transverse jet [Fig. 2(b)], jet strike [Fig. 2(c)], sheet jetting [Fig. 2(d)], and minimum volume [Fig. 2(e)] [11]. Given the highly nonlinear nature of the collapse during sheet jetting, we found the most robust method for comparing between simulations was to take a temporal average of the peak values of pressure, density, and temperature in the gas during jet strike. The window for this temporal average is defined as beginning when the on-axis distance between the jet and the leeward wall is constant until the point when the jet merges with the leeward wall and sheet jetting is initiated [Fig. 2(f)].

\section{RESULTS}

By comparing the structure of the gas-liquid interface during collapse for each argon EOS, we conclude that the processes prior to jet strike are governed by the characteristics of the liquid [Fig. 3(a)]. This is further corroborated by the negligible difference in the physical time of jet strike and the velocity of the jet at this point (result not shown), and the equivalent average peak pressures during jet strike which are invariant with regard to the gas EOS [Fig. 4(a)]. This matches expectations from previous work comparing gas and vapor cavities [46]. We find that conditions during jet strike form an objective measure and are an accurate proxy for the true peak conditions, which occur shortly before minimum volume. Beyond jet strike, the highly nonlinear nature of the collapse means that the shape of the gas-liquid interface diverges subtly; although not impacting on the bulk hydrodynamics [Fig. 3(b)], these changes make comparisons between different EOSs, particularly temperature, less robust. With that caveat in mind, we find that, for the $1000 \mathrm{GPa}$ incident shock, peak temperatures are four to eight times higher than the temperature at jet strike.

In the ideal gas study conducted by Hawker and Ventikos, a counterintuitive downward trend in the density during jet strike was discovered [11]. That study modelled air; we find the same feature when modeling argon by using a polytropic EOS [triangles in Fig. 4(b)]. We find that QEOS predicts the opposite, that density increases with shock strength, while SESAME predicts there to be a maximum in compression. These differences can be understood by examining the differences in the principal Hugnoiots. The multiple-shock compression present within the gas can be thought of in two steps; a single strong shock followed by quasi-isentropic compression from this once-shocked state [47]. As shown in Fig. 1, single shock can only compress to a limited extent. The temperature on the principal Hugoniot, however, continues 
(a) Time $=0.000 \mathrm{E}+00 \mathrm{~s}$

(b) Time $=2.080 \mathrm{E}-06 \mathrm{~s}$

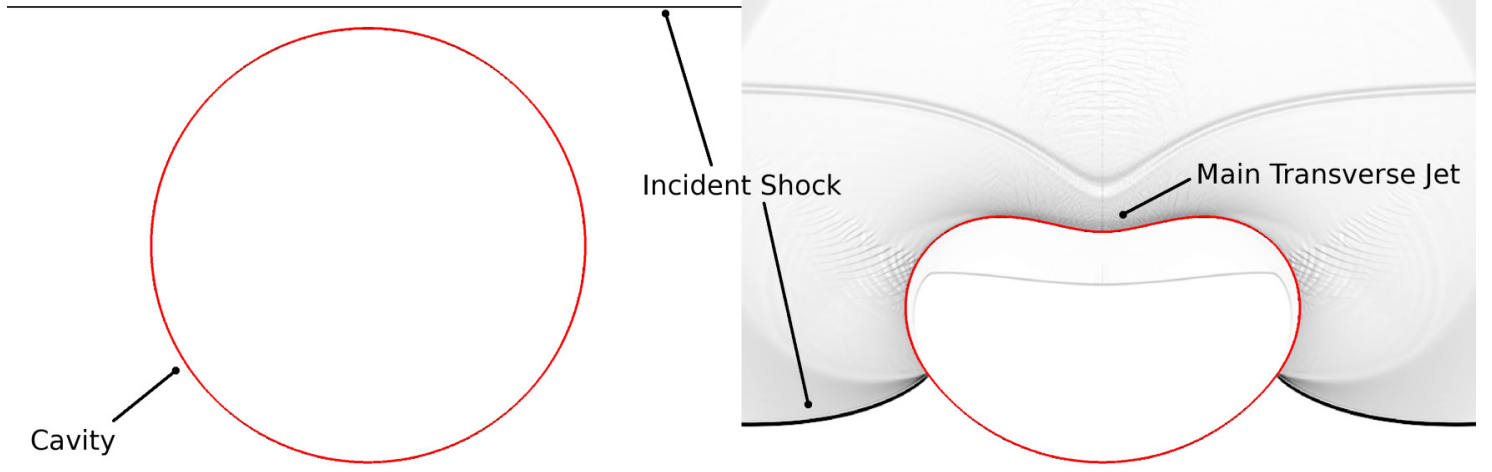

(c) Time $=3.449 \mathrm{E}-06 \mathrm{~s}$

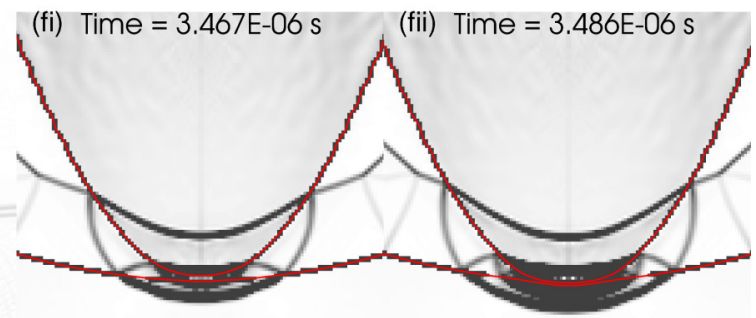

(fiii) Time $=3.504 \mathrm{E}-06 \mathrm{~s} \quad$ (fiv) Time $=3.522 \mathrm{E}-06 \mathrm{~s}$

(d) Time $=3.759 \mathrm{E}-06 \mathrm{~s}$

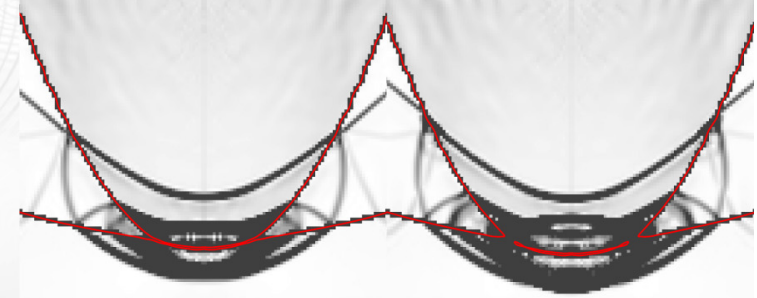

(e) Time $=4.179 \mathrm{E}-06 \mathrm{~s}$

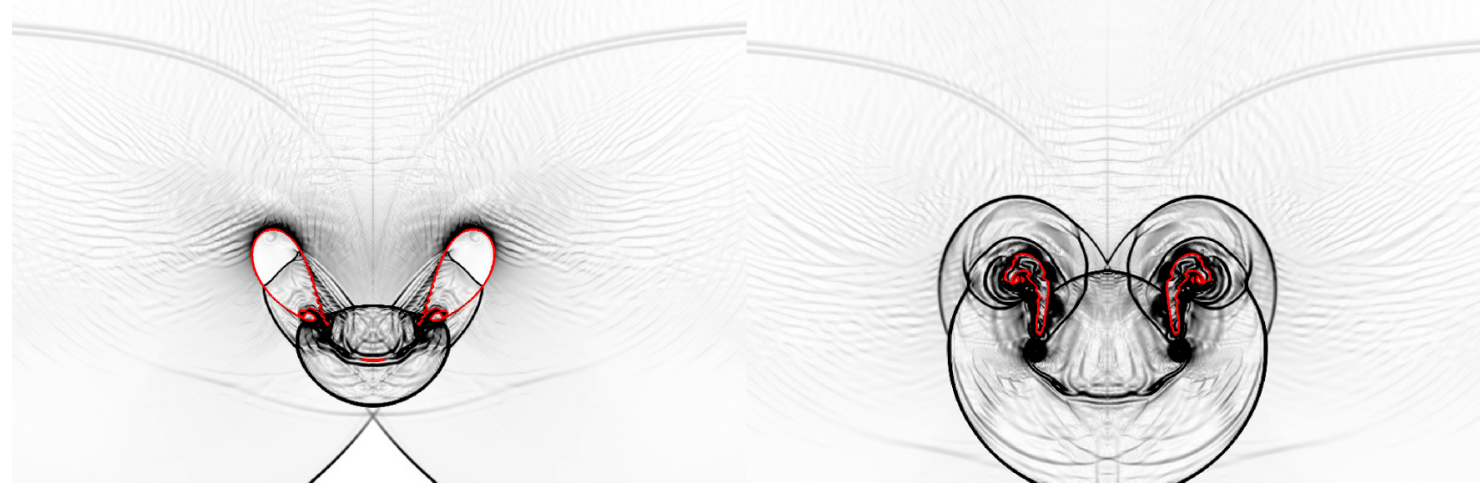

FIG. 2. Numerical schlieren, $n_{s}=|\nabla(\rho)|$, at various instances during the collapse with a $10 \mathrm{GPa}$ incident shock. The four inset images in panel (f) define the extent of the jet-strike event.

to rise. This affects the subsequent isentropic compression, where final density is correlated to initial density but inversely correlated to the initial temperature.

This balance of factors leads the principal Hugnoiot behavior of the different EOSs to be mapped to the compression seen within the cavity. At an incident shock pressure of $1 \mathrm{GPa}$, the first shock in the gas is $\sim 20 \mathrm{MPa}$. For polytropic, the principal Hugoniot has already approached maximum compression and the increasing temperature is thus the dominant effect, reducing the compression with increasing shock strength. 

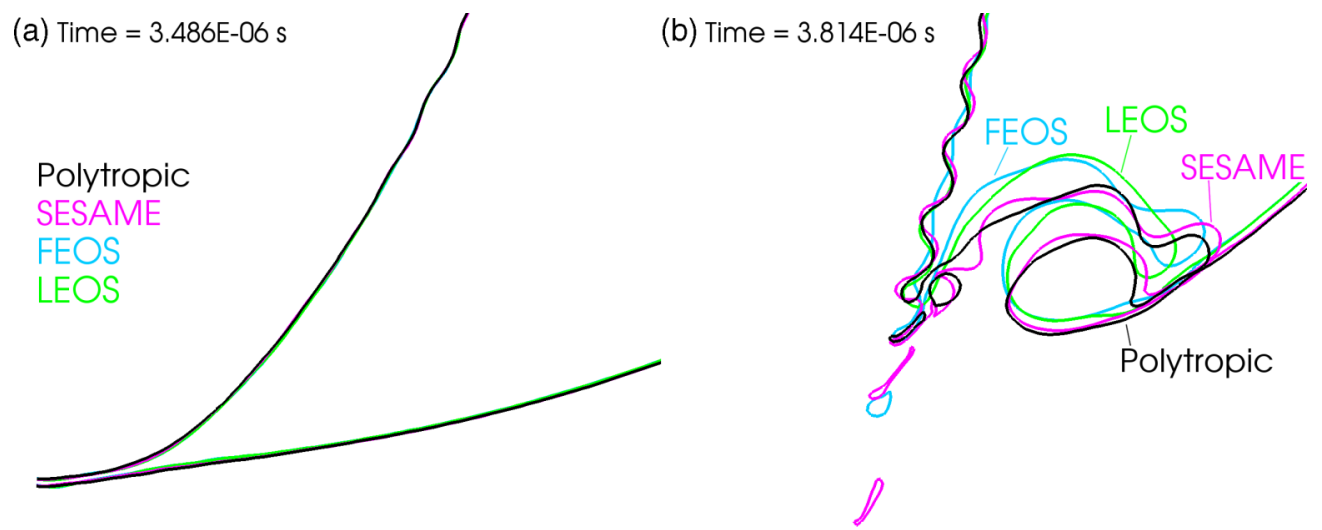

FIG. 3. A comparison of the gas-liquid interface for different EOS simulations with an incident shock strength $10 \mathrm{GPa}$.

QEOS shows the opposite behavior. The principal Hugoniot continuing to increase in compression until much larger

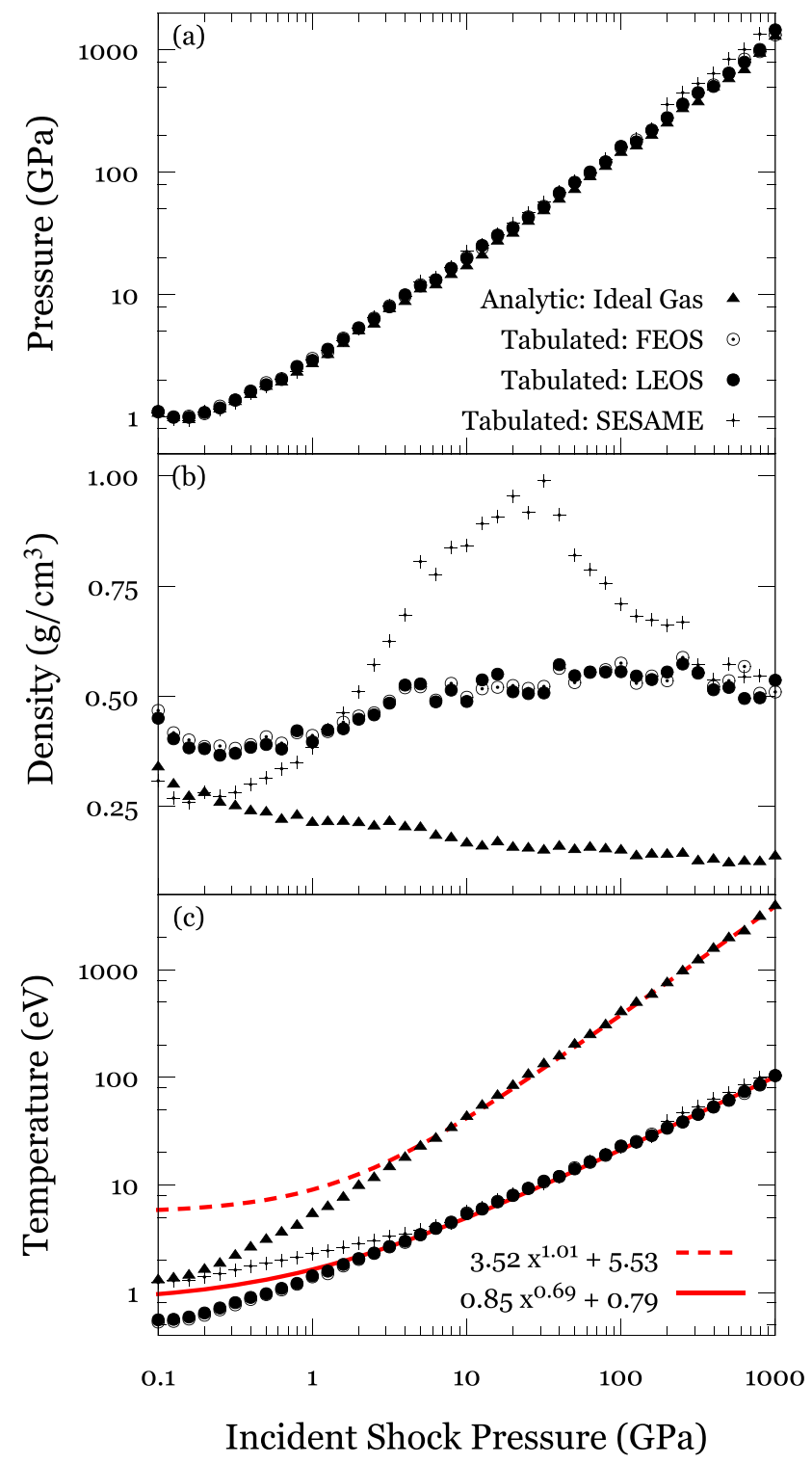

FIG. 4. Time averaged peak values of (a) pressure, (b) density, and (c) temperature during jet strike for the four different EOSs. pressures, meaning the additional compression in the first shock acts to counter the increased temperature and the final density increases with shock strength. SESAME conforms with expectations, demonstrating a peak in compression. These differences appear to the authors to make shock-driven cavity collapse experiments a possible source of new data for the testing and calibration of EOS.

Figure 4(c) shows the temperature during jet strike; the transition of SESAME between ideal gas and QEOS shown in the principal Hugoniot is also apparent here. At high shock strengths the ideal gas equation is clearly inappropriate while both families of plasma EOS converge to the same trend, albeit at reduced values. Importantly, the temperature remains a strictly increasing function of incident shock strength, approximately linear for ideal gas and a power law with exponent $\sim 0.7$ for both plasma EOS families. Through the use of PRISMSPECT (Prism Computation Sciences Inc, www.prism-cs.com) we estimate for the plasma EOS that both the average charge state and plasma parameter at jet strike reaches $\sim 1$ for an incident shock of $10 \mathrm{GPa}$, increasing to $\sim 10$ for the $1000 \mathrm{GPa}$ shock. We note, however, that various physical effects are not currently considered; for instance, at temperatures above $10 \mathrm{eV}$ radiative losses must be included for the results to be realistic.

\section{CONCLUSIONS}

Through the inclusion of a framework for tabulated equations of state, we have performed a detailed investigation on the effects of plasma physics modeling of gas during asymmetric cavity collapse. Conventional wisdom is that spherical collapse is necessary to achieve extreme conditions within a collapsing cavity [10], yet, we show conclusively that these conditions can be achieved with an asymmetric collapse given a sufficiently strong incident shock wave. By varying the pressure of the incident shock wave from 0.1 to $1000 \mathrm{GPa}$ for three families of argon EOSs, we find that the process of jet formation and the impact pressure are independent of EOS, while density is increased and temperature reduced by including plasma modeling. It is an acknowledged limitation of the current work that it is solving for Euler equations only; the current authors have shown that effects such as surface tension are not important for cavity-collapse simulations [11]; however, future work will introduce radiative transfer mechanisms because 
these are expected to influence results. With this caveat, we find that temperature remains a strictly increasing function of incident shock strength.

Thermodynamic properties of the warm-dense-matter regime are difficult to obtain experimentally [48]. We have shown that inertial collapse of a gas cavity by strong shock waves yields significant differences in predicted density between SESAME and QEOSs in this regime. These differences indicate that cavity collapse may provide a novel experimental method for exploring EOS data. Furthermore, the authors believe the direct relationship between input (incident shock strength) and output (temperature) is interesting for the technological application of shock-driven cavity collapse, for example, in material processing or chemical applications, as the required conditions can be achieved in a straightforward manner.

\section{ACKNOWLEDGMENTS}

We would like to thank David Young of Lawrence Livermore National Laboratory for his patience and guidance with the LEOS tables and comments on the manuscript. We gratefully thank Steffen Faik of Goethe University in Frankfurt for granting us access to the FEOS library.
[1] A. Thiruvengadam, Handbook of cavitation erosion, Tech. Rep. No. 7301-1 (Laurel, Md., Hydronautics, Inc., 1974), http://acwc.sdp.sirsi.net/client/en_US/default/search/ detailnonmodal/ent:\$002f\$002fSD_ILS\$002f0\$002fSD_ILS: 112610/ada/?qu=Cavitation.

[2] F. P. Bowden and O. A. Gurton, Nature (London) 161, 348 (1948).

[3] H. Frenzel and H. Schultes, Z. Phys. Chem. 27, 421 (1935).

[4] D. F. Gaitan, L. A. Crum, C. C. Church, and R. A. Roy, J. Acoust. Soc. Am. 91, 3166 (1992).

[5] C.-D. Ohl, O. Lindau, and W. Lauterborn, Phys. Rev. Lett. 80, 393 (1998).

[6] N. Bourne and J. Field, Philos. Trans. R. Soc., A 357, 295 (1999).

[7] W. Lauterborn and T. Kurz, Rep. Prog. Phys. 73, 106501 (2010).

[8] M. Brenner, S. Hilgenfeldt, and D. Lohse, Rev. Mod. Phys. 74, 425 (2002).

[9] T. Kurz, P. Koch, D. Schanz, and W. Lauterborn, J. Acoust. Soc. Am. 129, 2586 (2011).

[10] M. C. Ramsey and R. W. Pitz, Phys. Rev. Lett. 110, 154301 (2013).

[11] N. A. Hawker and Y. Ventikos, J. Fluid Mech. 701, 59 (2012).

[12] R. Nourgaliev, T. N. Dinh, and T. G. Theofanous, J. Comput. Phys. 213, 500 (2006).

[13] C.-H. Chang and M.-S. Liou, J. Comput. Phys. 225, 840 (2007).

[14] C. Hirt and B. Nichols, J. Comput. Phys. 39, 201 (1981).

[15] J. A. Sethian, Level Set Methods and Fast Marching Methods (Cambridge University Press, Cambridge, UK, 1999).

[16] J. Glimm, J. J. W. J. Grove, X. X. L. Li, and N. Zhao, Contemp. Mat. 238, 133 (1999).

[17] J. Glimm, J. W. Grove, X. L. Li, K.-m. Shyue, Y. Zeng, and Q. Zhang, SIAM J. Sci. Comput. 19, 703 (1998).

[18] J. Glimm, J. W. Grove, X. L. Li, and D. C. Tan, SIAM J. Sci. Comput. 21, 2240 (2000).

[19] B. van Leer, J. Comput. Phys. 32, 101 (1979).

[20] P. Colella, SIAM J. Sci. Stat. Comp. 6, 104 (1985).

[21] E. F. Toro, M. Spruce, and W. Speares, Shock Waves 4, 25 (1994).

[22] R. Menikoff and B. J. Plohr, Rev. Mod. Phys. 61, 75 (1989).

[23] R. M. More, K. H. Warren, D. A. Young, and G. B. Zimmerman, Phys. Fluids 31, 3059 (1988).

[24] S. P. Lyon and J. D. Johnson, Sesame: Los Alamos National Laboratory Equation of State Database, Los Alamos Report No. LA-UR-92-3407, 1992 (unpublished).

[25] E. H. Lieb and B. Simon, Adv. Math. 23, 22 (1977).
[26] S. Faik, M. M. Basko, A. Tauschwitz, I. Iosilevskiy, and J. A. Maruhn, High Energy Density Phys. 8, 349 (2012).

[27] A. Kemp and J. Meyer-ter Vehn, Nucl. Instrum. Methods Phys. Res., Sect. A 415, 674 (1998).

[28] D. A. Young and E. M. Corey, J. Appl. Phys. 78, 3748 (1995).

[29] S. Gewurtz and B. Stoicheff, Phys. Rev. B 10, 3487 (1974).

[30] E. R. Dobbs and G. O. Jones, Rep. Prog. Phys. 20, 516 (1957).

[31] D. A. Young, High Pressure Res. 16, 389 (2000).

[32] J. Wolford and K. Long, Tech. Rep. No. UCID-18574-81-3, Lawrence Livermore National Laboratory, 1981 (unpublished).

[33] C. H. Hodges, Can. J. Phys. 51, 1428 (1973).

[34] O. K. Andersen, Phys. Rev. B 12, 3060 (1975).

[35] M. Ross, J. Chem. Phys. 71, 1567 (1979).

[36] J. E. Jones, Proc. R. Soc. London, Ser. A 106, 463 (1924).

[37] R. H. Christian and F. L. Yarger, J. Chem. Phys. 23, 2042 (1955).

[38] J. Carpenter, S. Root, and K. Cochrane, Tech. Rep. No. SAND2012-7991, August, Sandia National Laboratories, Sandia, 2012 (unpublished).

[39] D. E. Maiden, in 1998 Nuclear Explosives Development Conference (Lawrence Livermore National Laboratory, Livermore, CA, Las Vegas, NV, 1998).

[40] B. J. Plohr, AIAA J. 26, 470 (1988).

[41] G. I. Kerley, Tech. Rep. No. LA-6903-MS, Los Alamos National Laboratory, Los Alamos, 1977 (unpublished).

[42] R. E. Carlson and F. N. Fritsch, SIAM J. Numer. Anal. 26, 230 (1989).

[43] F. N. Fritsch, Tech. Rep. No. LLNL-TR-406719-REV-3, Lawrence Livermore National Laboratory, Livermore, 2011 (unpublished).

[44] B. Tully, N. Hawker, M. Betney, and Y. Ventikos, Proc. Mtgs. Acoust. 19, 075040 (2013).

[45] M. R. Betney, B Tully, N. A. Hawker, and Y. Ventikos, Phys. Fluids 27, 036101 (2015).

[46] Y. Jinbo and H. Takahira, in 19th International Symposium on Nonlinear Acoustics State-of-the-Art and Perspectives, edited by T. Kamakura and N. Sugimoto, AIP Conf. Proc. No. 1474 (American Institute of Physics, New York, 2012), pp. 139-142.

[47] W. J. Nellis, S. T. Weir, and A. C. Mitchell, Phys. Rev. B 59, 3434 (1999).

[48] D. A. Young, in Workshop on Accelerator-Driven Warm Dense Matter Physics (The Heavy Ion Fusion Virtual National Laboratory, Pleasanton, CA, 2006). 